\title{
CÁLCULO DA QUANTIDADE ÓTIMA DE RESSUPRIMENTO PARA MINIMIZAÇÃO DO CUSTO DE MANUTENÇÃO DO ESTOQUE DE ITENS DO SISTEMA DE ABASTECIMENTO DA MARINHA
}

\author{
Alan Matias Avelar \\ Diretoria de Abastecimento da Marinha \\ e-mail: alan.matias@dabm.mar.mil.br \\ Thiago Fernandes Lima \\ Diretoria de Abastecimento da Marinha \\ e-mail: fernandes.lima@dabm.mar.mil.br \\ Lenise Moreira Ribeiro \\ Diretoria de Abastecimento da Marinha \\ e-mail: lenise@dabm.mar.mil.br \\ David Barros Maia \\ Diretoria de Abastecimento da Marinha \\ e-mail: david.barros@dabm.mar.mil.br
}

\begin{abstract}
Resumo
Com intuito de minimizar o custo de manutenção de estoque de itens do Sistema de Abastecimento da Marinha (SAbM), um simulador foi desenvolvido em MS Excel/VBA e aplicado para análise de modelos de determinação do estoque de segurança e otimização do custo de estocagem de materiais de uma mesma categoria e classe. Os resultados obtidos foram comparados com séries históricas obtidas do Sistema de Informações Gerenciais de Abastecimento da Marinha (SINGRA), e demonstram que o modelo pode ser aplicado para redução do custo de manutenção de estoque, por meio de uma visão sistêmica do abastecimento.

Palavras-Chaves: $\quad$ Abastecimento; Otimização; Custo de manutenção de estoque.
\end{abstract}

Abstract
In order to minimize the cost of holding inventory of items from the Brazilian Navy Supply System, a simulator was developed in MS Excel / VBA and applied to analyze models of determining the safety inventory and also, to optimize the cost of holding inventory of items from same category and class. The results were compared to historical data obtained from SINGRA Management System, and demonstrate that the model can be applied to reduce the cost of holding inventory, through a systemic vision of supply.

Keywords: $\quad$ Supply; Optimization; Cost of holding inventory.

\section{INTRODUÇÃO}

O Sistema de Abastecimento da Marinha (SAbM) tem a finalidade de contribuir para prever e prover o material necessário, a fim de manter os meios operativos e demais organizações militares da Marinha do Brasil em condições de plena eficiência, proporcionando um fluxo adequado do material, desde as fontes de obtenção, até as organizações militares consumidoras. O gerenciamento das diferentes cadeias de suprimentos do SAbM compõem um problema logístico complexo, de controle de inventário e de fluxos, de diversas categorias de materiais. [6][7] O controle de inventários é uma preocupação importante nesse sistema, pois, há nele o desafio da redução de custos de estocagem e 
satisfação de necessidades, de itens com demanda obrigatória, incerta e sazonal; e com diferentes níveis de complexidade tecnológica.

A solução logística necessária à eficiência do SAbM, parte sobremaneira da eficácia das ações e metodologias implementadas no planejamento do abastecimento. De maneira que, questões como orçamento e espaço de armazenamento, compõem restrições que podem prejudicar a eficiência do abastecimento, no caso de um planejamento que não tenha antecipado discussões acerca desses limiares. Nesse sentido, o planejamento para a minimização de estoques, aliada à manutenção da disponibilidade de orçamento, podem contribuir incisivamente para o aumento da eficiência do abastecimento. [7]

De fato, a principal razão para manter os estoques em um nível reduzido, é que o estoque representa um investimento monetário temporário em bens que geram custos ao sistema. Entende-se por custo de manutenção do estoque, o custo variável de manter itens disponíveis, incluindo custos de oportunidade, armazenagem, manuseio, impostos, seguros e perdas. [3][6][14][15][16] Entende-se por custo de oportunidade, o preço da renuncia de um bem de modo a se obter outro bem. [2][9][14][15] Nesse contexto, a seleção do modelo de determinação do estoque de segurança deve ser considerada, pois, além de influenciar o ponto de recolocação do pedido, determina uma importante componente do custo de estocagem.

Com intuito de minimizar o custo de manutenção de estoque, um simulador foi desenvolvido em MS Excel/VBA/Solver e aplicado para análise de modelos de determinação do estoque de segurança e otimização do custo de estocagem de materiais de uma mesma categoria e classe. Os resultados obtidos foram comparados com séries históricas obtidas do Sistema de Informações Gerenciais de Abastecimento da Marinha (SINGRA), e demonstram que o modelo pode ser aplicado para redução do custo de manutenção de estoque, por meio de uma visão sistêmica do abastecimento.

\section{SISTEMAS DE CONTROLE DE ESTOQUE}

A função principal do estoque é de garantir a disponibilidade do produto ao consumidor e, conforme enfatizado por Slack et al. (2010) [11] por ser definido de forma simplória como um recurso armazenado. Como qualquer recurso, o estoque deve ser administrado. A administração de estoques, seu planejamento e seu controle a fim de satisfazer às prioridades competitivas da organização é um assunto importante para gerentes em todos os tipos de negócios. A gestão eficaz de estoques é essencial para concretizar o potencial pleno de qualquer cadeia de valor. [6][15][16]

Ao longo dos anos, a visão das empresas e organizações em relação à forma de lidar com a gestão de estoques têm evoluído bastante. Os estoques eram inicialmente utilizados como elemento-chave no balanceamento da cadeia de suprimentos (grande quantidade de material parado ao longo da cadeia), ou seja, funcionavam como um pulmão para a proteção contra as ineficiências do processo. Neste enfoque clássico, temos, por exemplo, a abordagem do Lote Econômico de Compras (LEC), onde os estoques eram renovados de forma a minimizar a soma do custo do inventário, do custo de transporte e do custo para elaborar o pedido. Tal comportamento era respaldado pela falta de diversidade de produtos e ao mesmo tempo a falta de competitividade. [3][6][13][14]

O crescimento da variedade de produtos e a mudança de perfil do consumidor típico, que passou a aspirar produtos diferenciados, gradativamente influenciaram a forma como as organizações passaram a enxergar as atividades logísticas de forma estratégia e não mais como meras geradoras de custos. Elevando, assim, as atividades desenvolvidas nos diferentes segmentos da logística: inbound (logística de suprimento), intralogística (logística interna) e outbound (logística de distribuição), como fatores de alavancagem competitiva e, consequentemente, de sobrevivência no mercado. [3][14]

As decisões relacionadas à gestão de estoques possuem grande impacto nos custos decorrentes das atividades que serão desenvolvidas nos diferentes segmentos da logística 
citados acima, bem como nos níveis de serviço ao cliente. Logo, as decisões de quanto e quando pedir, devem ser apoiadas por políticas de gestão de estoques e ferramentas de apoio a decisão adequadas. Segundo Wanke (2011) [14], tem-se dois elementos de definição que suportam à decisão entre reagir à demanda (pull) ou planejar com base em previsão (push), são eles: a visibilidade da demanda e o tempo de resposta, também chamados de características da operação. Em situações em que não há visibilidade total da demanda e os tempos de respostas não são curtos as decisões de quanto e quando pedir deve ser baseadas na antecipação da quantidade em relação ao consumo real. A figura 1 apresenta um modelo de gestão de estoque genérico, com a nomenclatura utilizada neste trabalho.

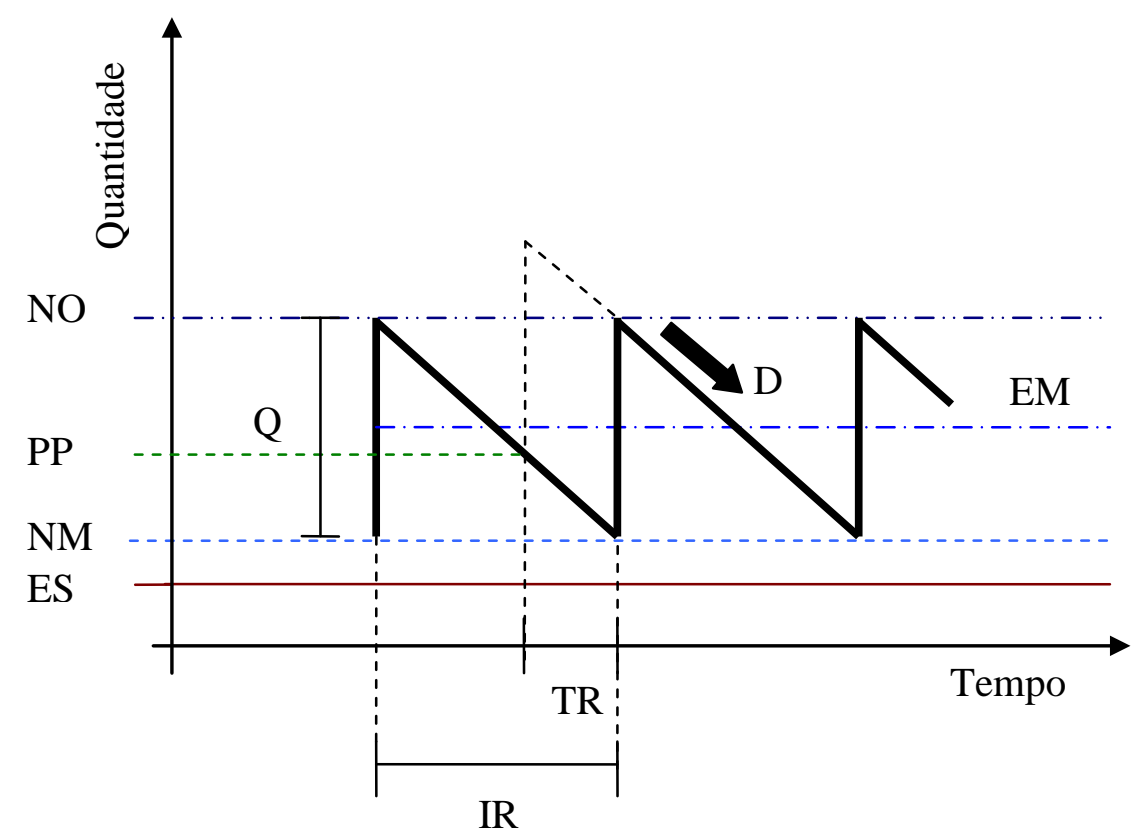

Figura 1 - Exemplo de um modelo de controle de estoque. Adaptado de Silva (2006) [12] Nomenclatura: D - Demanda; EM - Estoque Médio; ES - Estoque de Segurança; IR Intervalo de ressuprimento; NM - Nível Mínimo; NO - Nível de Operação; PP - Ponto do Pedido; Q - Quantidade de ressuprimento; e TR - Tempo de resposta.

A despeito das diferenças entre os modelos de determinação de estoque de segurança disponíveis na literatura. [1][3][6][14][15], é comum para itens de alta demanda, a abordagem com probabilidade de distribuição Normal, especificada por média e desvio-padrão da demanda durante o tempo de resposta, conforme apresentado na figura 2:

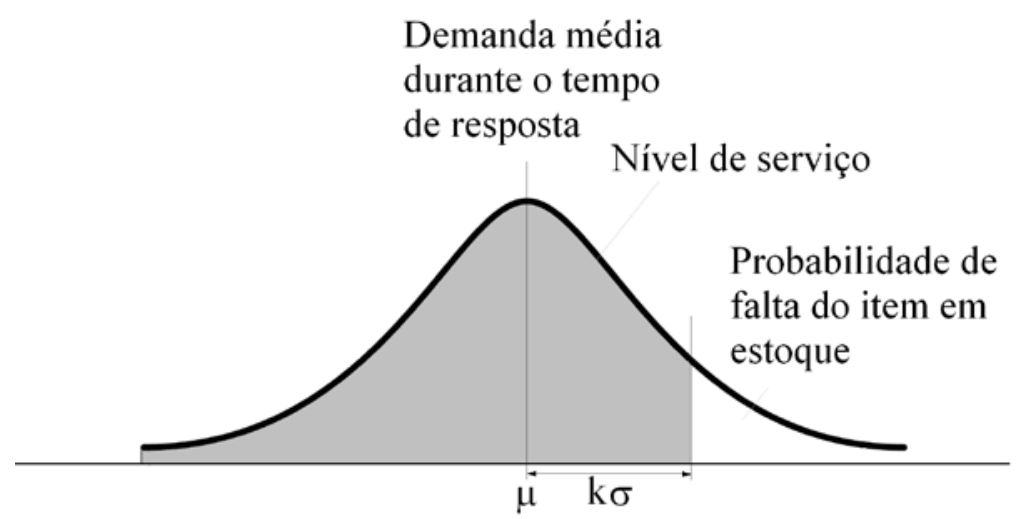

Figura 2 - Nível de serviço e probabilidade de faltar o item em estoque durante o tempo de resposta. Adaptado de Krajewski et al. (2009) [6]. 


\section{METODOLOGIA}

\subsection{AVALIAÇÃo do MODELO DE DETERMINAÇÃo do ESTOQUE DE SEGURANÇA}

Um dos modelos sugeridos por Wanke (2011) [14] para operações de múltiplos períodos foi utilizado para avaliar, por meio de comparação, a atual formulação de estoque de segurança contida no Sistema de Informações Gerenciais de Abastecimento (SINGRA). A análise foi conduzida para "n” itens, de alta demanda e de uma mesma categoria de material; ao longo de um período de tempo "m", sendo “m” igual a 13 (treze) trimestres, compreendidos entre o último trimestre de 2009 e o último trimestre de 2012. O modelo, descrito na equação 1 considera, além da probabilidade de não faltar produto, a variabilidade da demanda no tempo de resposta e a distribuição de probabilidade da demanda no tempo de resposta, haja vista a suscetibilidade de faltas apenas no intervalo compreendido entre o momento de colocação do pedido e o momento de recebimento do mesmo. [14][15]

$$
E S=k \times \sqrt{T R \times \square_{D}^{2}+D^{2} \times \square_{T R}^{2}}
$$

Sendo:

ES - Estoque de segurança;

$k$ - Quantidade de desvios-padrão para um determinado nível de serviço;

$T R$ - Média do tempo de resposta;

$\square_{R}$ - Desvio-padrão do tempo de resposta;

$D$ - Média da demanda durante o tempo de resposta; e

$\square$ - Desvio-padrão da demanda durante o tempo de resposta.

Com intuito de calcular o estoque de segurança dos itens, as séries temporais de demanda e tempo de resposta, foram extraídas do Sistema de Informações Gerenciais de Abastecimento (SINGRA). Conforme sugerido por Wanke (2011) [14] os cálculos de média e desvio-padrão da demanda foram efetuados considerando a série temporal da demanda somente durante o período do tempo de resposta referente ao trimestre imediatamente anterior. Por outro lado, os cálculos de média e desvio-padrão do tempo de resposta foram efetuados considerando a série temporal de tempo de resposta do ano imediatamente anterior.

\subsection{OTIMIZAÇÃo DO CUSTO DE MANUTENÇÃO DO ESTOQUE}

\subsubsection{Função objetivo}

O custo de manutenção de estoque é uma componente da função custo total utilizada no Lote Econômico de Compras (LEC). [1][3][6][14] Para uma análise logística inbound, mais especificamente no que se refere à entrada de materiais em locais de armazenagem da Marinha do Brasil, desconsidera-se custos com transporte e colocação de pedidos, de tal forma que, apenas o custo total de manutenção de estoque é definido como função objetivo.

O vetor custo de manutenção de estoque consiste da diagonal principal do produto da matriz custo unitário de manutenção em estoque pela transposta da matriz estoque médio, conforme a equação 2:

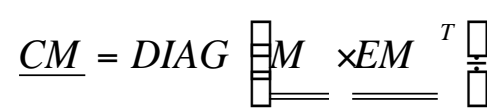

Sendo:

$\underline{C M}$ - Vetor custo de manutenção de estoque (m Trimestres x 1). 

Produtos); e

$\underline{\underline{M}}$ - Matriz custo unitário de manutenção em estoque em reais (m Trimestres x n $\underline{\underline{E M^{T}}}$ - Transposta da matriz estoque médio (n Produtos x m Trimestres).

A matriz estoque médio é composta pelos valores do estoque médio dos itens nos intervalos de ressuprimento, sendo calculada como a média dos níveis de operação e mínimo.

A matriz custo unitário de manutenção em estoque, descrita na equação 3 , apresenta os custos unitários de se manter determinado item em estoque. O cálculo desse custo é composto pelo produto entre o seu custo de aquisição e uma taxa de oportunidade. Neste trabalho utilizou-se a taxa do Sistema Especial de Liquidação e de Custódia (SELIC) [5][9] do trimestre, como a taxa de oportunidade do capital, pois, é a taxa de juros referencial para títulos federais. Para ajustar o histórico dos preços praticados no mercado conforme a inflação do período, os custos de aquisição dos materiais para os períodos foram descapitalizados, utilizando o Índice Nacional de Preços ao Consumidor (IPCA) [4]. De tal forma que, o custo de aquisição do item é igual ao seu custo no período posterior $(\mathrm{m}+1)$, descapitalizado com o IPCA do trimestre em análise. Como os dados das taxas são disponibilizados mensalmente, $\mathrm{O}$ cálculo da taxa equivalente foi realizado em ambas os casos, com juros compostos, para determinação das taxas correspondentes a um período trimestral.

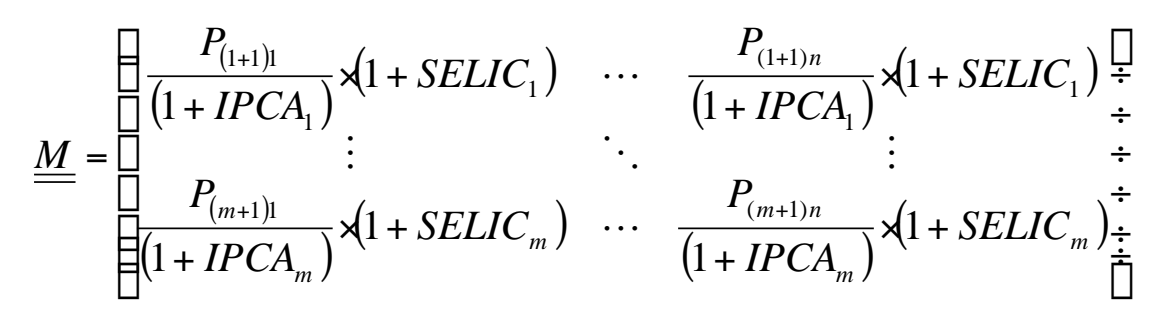

Sendo:

$P_{(j+1) i}$ - Preço do item i no período $(\mathrm{j}+1)$ em reais $(\mathrm{i}=1, \ldots, \mathrm{n}$ e $\mathrm{j}=1, \ldots, \mathrm{m})$; período m; e

$I P C A_{m}$ - Taxa equivalente ao Índice Nacional de Preços ao Consumidor (IPCA) no

$S E L I C_{m}$ - Taxa equivalente a do Sistema Especial de Liquidação e de Custódia (SELIC) no período $\mathrm{m}$.

Utilizando as equações 2 e 3, o vetor custo de manutenção de estoque pode ser escrito conforme a equação 4:

Sendo:

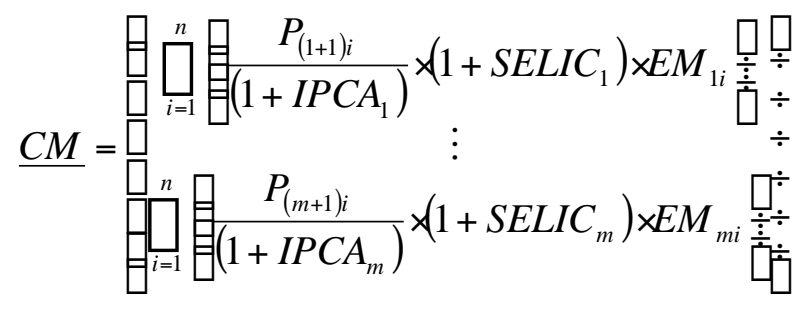

$E M_{j i}$ - Estoque médio do item i no j-ésimo período.

Para um dado período, o custo total de manutenção de estoque é dado pelo somatório das componentes trimestrais do vetor custo de manutenção de estoque, conforme a equação conforme a equação 5: 


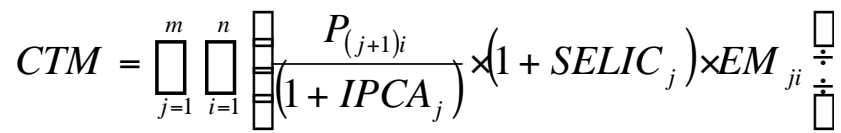

Sendo:

$C T M$ - Custo total de manutenção do estoque em reais; e

\subsubsection{Restrições e Variáveis de Decisão}

\subsubsection{Orçamento}

A primeira restrição condiciona que as execuções financeiras correspondentes às obtenções dos períodos, sejam menores ou iguais aos orçamentos disponíveis para os períodos, conforme expresso na inequação 6:

\section{$\underline{E X} \square \underline{O R}$}

Sendo:

$\underline{E X}$-Vetor execução financeira referente às obtenções trimestrais em reais (m Trimestres X 1); e

$\underline{O R}$ - Vetor orçamento em reais (m Trimestres $\mathrm{X} 1$ ).

O vetor execução financeira é dado pela diagonal principal do produto entre a matriz quantidade de ressuprimento e a transposta da matriz preço, conforme expresso na equação 7:

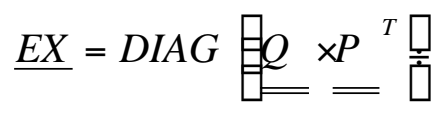

Sendo:

$\underline{\underline{Q}}$ - Matriz quantidade de ressuprimento (m Trimestres X n Produtos); e

$\underline{\underline{P}}^{T}$ - Transposta da matriz preço unitário em reais (n Produtos X m Trimestres).

\subsubsection{Espaço}

A segunda restrição limita o espaço disponível para estocagem de materiais em um determinado depósito. Estimou-se o espaço de armazenamento máximo disponível para esses itens, em 20\% da área útil de um depósito de 140 x 70 metros, com pé direito de 10 metros. Essa restrição está formulada conforme a inequação 8:

$$
\underline{V O} \square \underline{V D}
$$

Sendo:

$\underline{V O}$-Vetor volume total ocupado em $\mathrm{m}^{3}$ (m Trimestres $\mathrm{X} 1$ ); e

$\underline{V D}$-Vetor volume disponível em $\mathrm{m}^{3}$ (m Trimestres $\mathrm{X} 1$ ).

Sendo o volume total ocupado calculado no nível de operação de estoque restaurado, ou seja, no ponto de recebimento do pedido. Desse modo, calcula-se o vetor volume total ocupado como sendo a diagonal principal do produto matricial entre a matriz nível de operação e a transposta da matriz volume unitário, conforme a equação 9: 


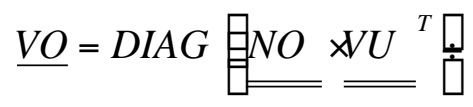

Sendo:

$\underline{\underline{N O}}$ - Matriz nível de operação do estoque (m Trimestres X n Produtos); e

$\underline{\underline{V U^{T}}}$-Transposta da matriz volume da unidade de fornecimento do produto em $\mathrm{m}^{3}$ (n Produtos X m Trimestres).

O nível de operação de um determinado produto em um dado período é calculado como sendo o somatório dos ressuprimentos efetuados até o presente período, acrescido da quantidade inicial em estoque e subtraído do somatório das demandas efetuadas até o período imediatamente anterior.

\subsubsection{Estoque de Segurança}

Outra restrição, aplicável a otimização reside no fato de que a quantidade de material disponível em estoque no instante imediatamente anterior ao ressuprimento, não deve ser inferior ao estoque de segurança, calculado por meio da equação 1. Matematicamente, essa restrição se expressa conforme a equação 10:

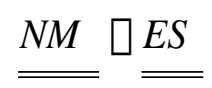

Sendo:

$\underline{\underline{E S}}$ - Matriz estoque de segurança (m Trimestres X n Produtos); e

$\underline{\underline{N M}}$ - Matriz nível mínimo de estoque (m Trimestres $\mathrm{X}$ n Produtos).

O nível mínimo de um determinado produto em um dado período é calculado como sendo o somatório dos ressuprimentos efetuados até o presente período, acrescido da quantidade inicial em estoque e subtraído do somatório das demandas efetuadas até o período.

\subsubsection{Formulação do problema}

Desse modo, o problema de otimização foi estruturado da seguinte forma:

Problema: MIN CTM

Variáveis de decisão: $\stackrel{Q}{=}$

Restrições: $\quad \underline{E X} \square \underline{O R}$

$$
\begin{aligned}
& \frac{V O}{N M} \underline{V D} \\
& \square E S
\end{aligned}
$$

\section{RESULTADOS E DISCUSSÕES}

\subsection{AVALIAÇÃO DO MODELO DE DETERMINAÇÃO DO ESTOQUE DE SEGURANÇA}

O modelo de determinação de estoque de segurança descrito pela equação 1 foi aplicado para o mesmo nível de serviço, estabelecido pelo modelo constante no Sistema de Informações Gerenciais de Abastecimento (SINGRA), para a categoria de material analisado. Sendo o nível de serviço igual a 85\%, tem-se que a quantidade de desvios-padrão é igual a 1,04. As figuras 3 e 4 apresentam os resultados obtidos em dois exemplos de itens analisados, 190000006 e 190011571 , respectivamente. 


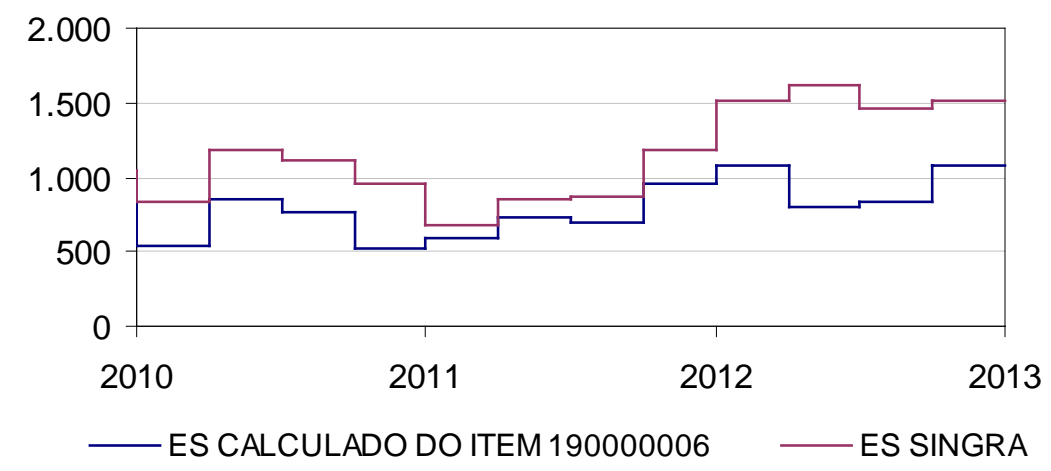

Figura 3 - Comparação entre o estoque de segurança calculado e os dados obtidos com o atual modelo do SINGRA, para o item 190000006.

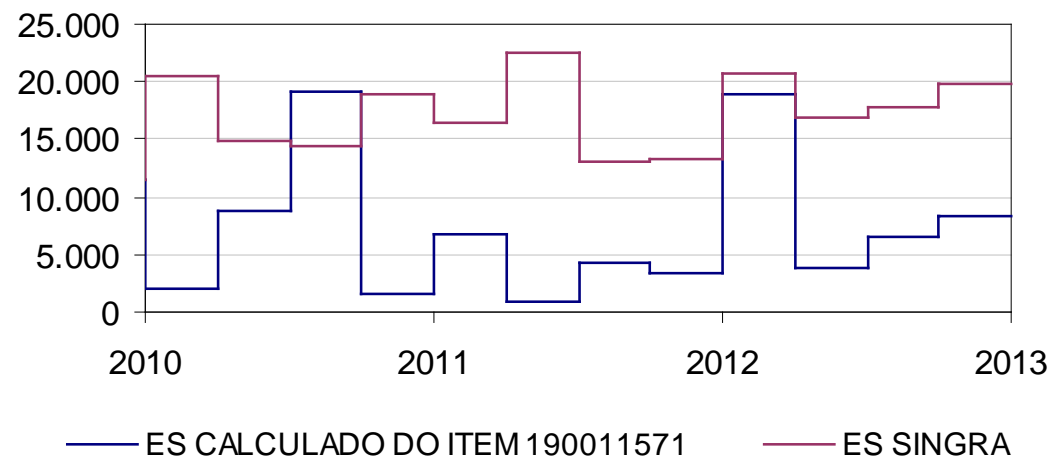

Figura 4 - Comparação entre o estoque de segurança calculado e os dados obtidos com o atual modelo do SINGRA, para o item 190011571.

Os resultados demonstram que, para um mesmo nível de serviço, o modelo sugerido por Wanke (2011) [14] proporciona um nível de estoque de segurança inferior ao obtido pelo modelo atualmente implementado no SINGRA. Observa-se também, que o modelo calculado responde as oscilações da demanda com maior rapidez e intensidade. Ambos os resultados, decorrem do fato que o modelo calculado utiliza a série temporal da demanda, apenas durante o tempo de resposta. Por outro lado, o modelo implementado considera quatro períodos anteriores, o que lhe confere maior estabilidade e um resultado mais conservativo.

\subsection{OTIMIZAÇÃO DO CUSTO DE MANUTENÇÃO DO ESTOQUE}

Uma vez executada a rotina de otimização, as quantidades de ressuprimento foram otimizadas, minimizando o custo total de manutenção de estoque; garantindo todas as restrições impostas. O estoque otimizado para um dos itens analisados encontra-se na figura 5: 


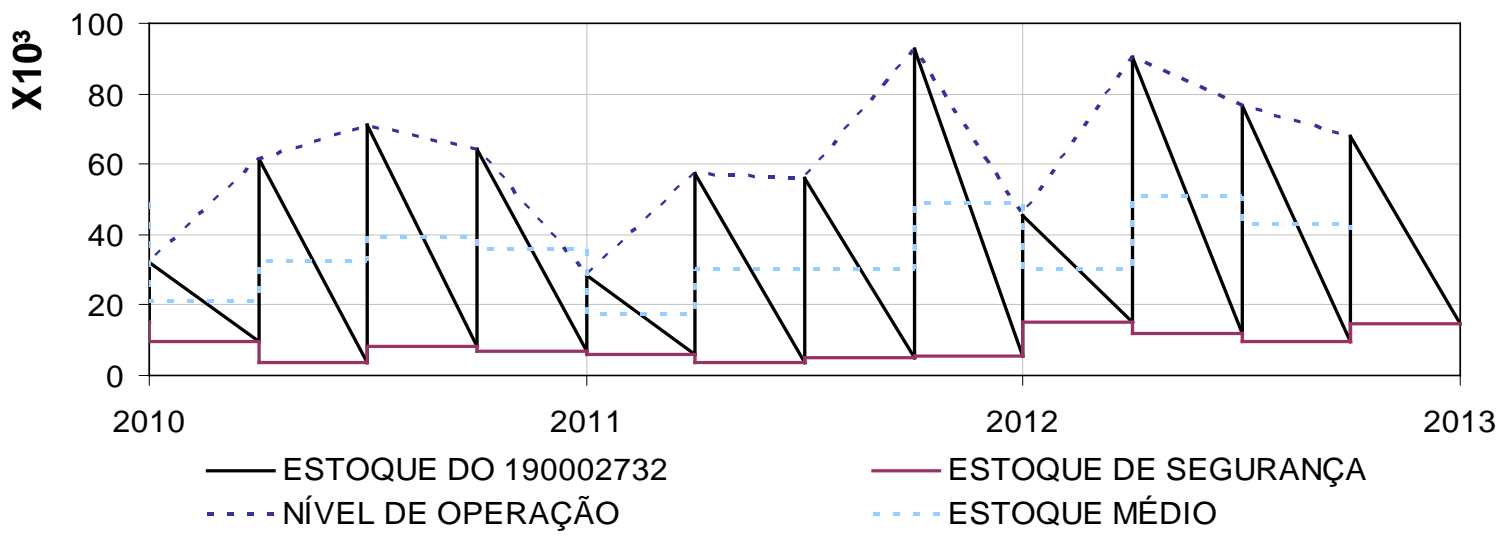

Figura 5 - Resultado do controle de estoque otimizado para o item 190002732.

Com relação ao custo de manutenção de estoque, observa-se, conforme representado na figura 6 , que o custo otimizado representa em média, $46 \%$ do custo realizado. Caso essa economia fosse aplicada à uma taxa de juros equivalente a SELIC [5], resultaria em um saldo de cerca de $\mathrm{R} \$ 20$ milhões de reais, no final do período analisado.

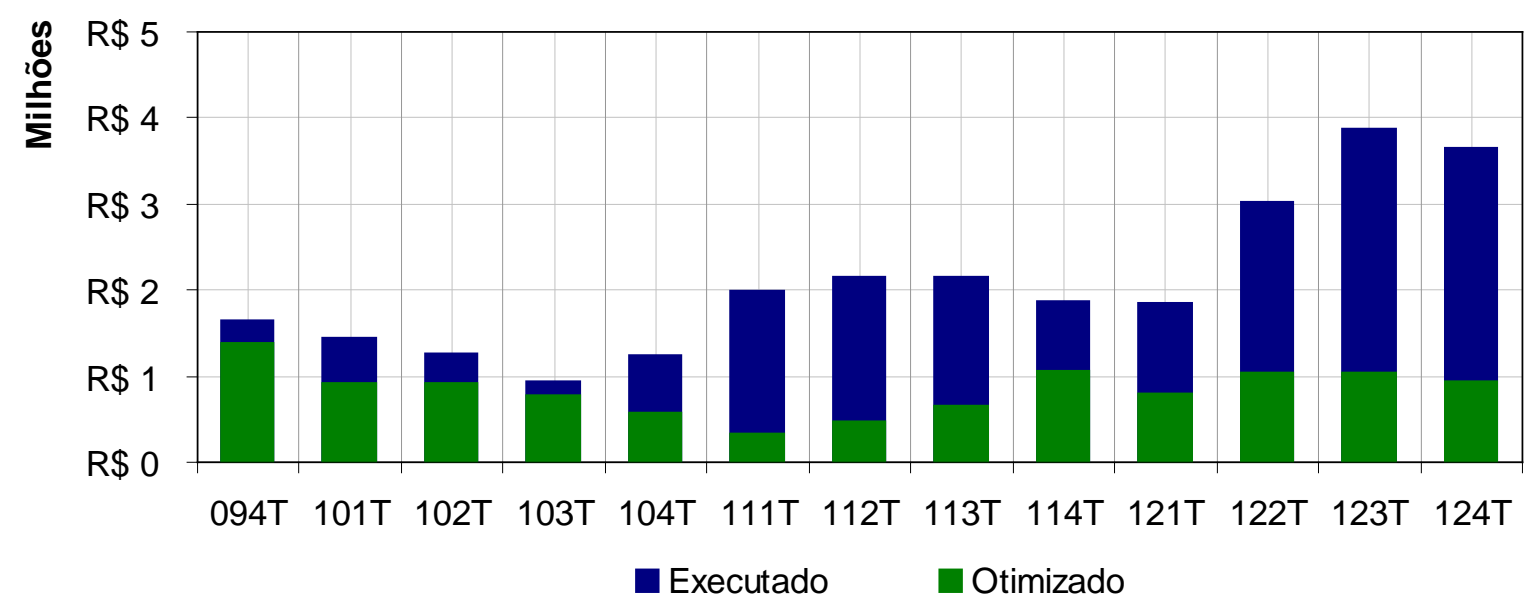

Figura 6 - Custo de manutenção de estoque otimizado e executado.

As restrições de orçamento e volume foram atendidas. Ressaltam-se alguns instantes, em que houve a necessidade de obtenções otimizadas com valores superiores às executadas. Entretanto, de forma geral, o dispêndio financeiro otimizado é cerca de $90 \%$ do executado. Conforme se observa na figura 7: 


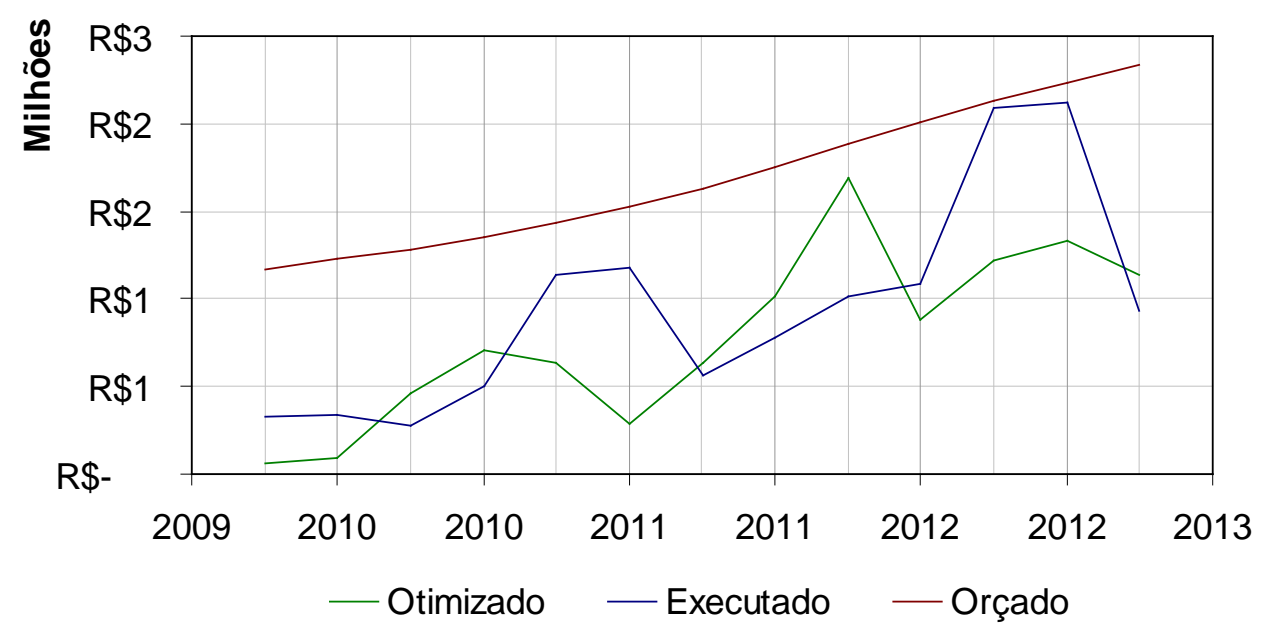

Figura 7 - Restrição orçamentária, execução financeira realizada e simulação de execução com otimização do custo de estocagem.

No que diz respeito ao espaço, o resultado da otimização proporcionou uma ocupação média de 38\% do espaço utilizado durante a execução. Conforme se observa nas figuras 8 (a) e (b), há uma redução da escala do volume ocupado.

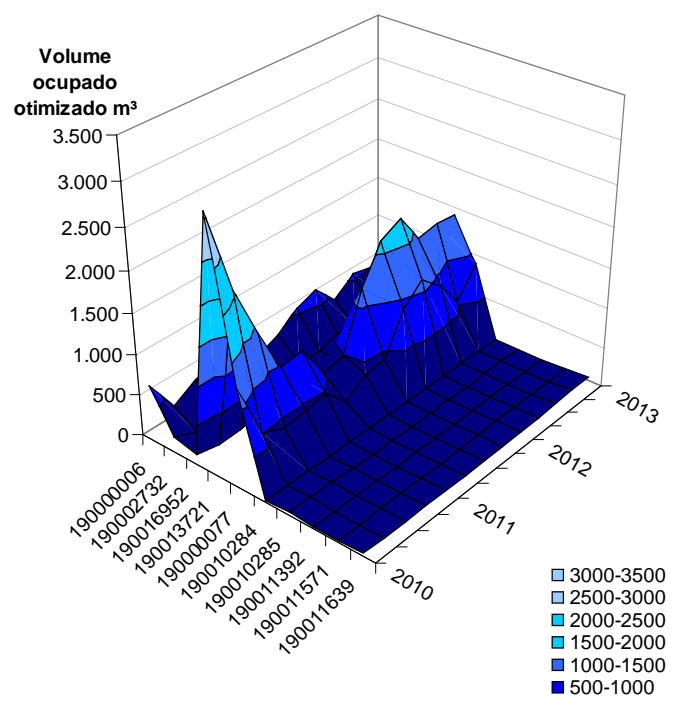

(a)

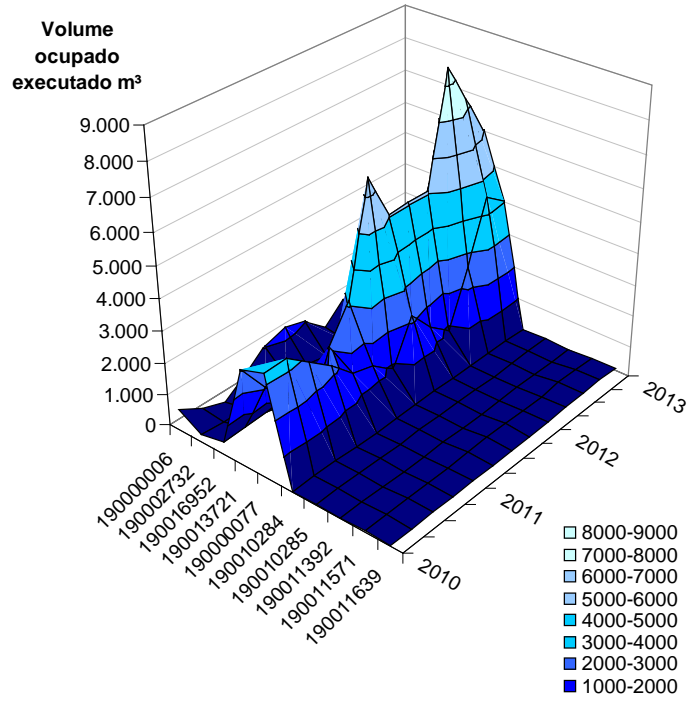

(b)

Figura 8 - Volume $\left(\mathrm{m}^{3}\right)$ ocupado no depósito por tipo de material e por período (a) otimizado e (b) executado.

\section{CONCLUSÕES}

A economia proporcionada pela redução dos custos de estocagem, aplicada a uma taxa de juros equivalente à taxa de oportunidade, é capaz prover recursos valiosos, haja vista a suscetibilidade de cortes orçamentários do governo. A disponibilidade deste recurso abre espaço para que aquisições mais eficientes possam ser conduzidas.

A opção por modelos menos conservativos de determinação de estoque de segurança, que consideram a demanda no tempo de resposta aderente à distribuição Normal, pode ser 
avaliada para itens julgados como não críticos e com padrão de demanda de alto consumo. Uma vez que, o nível de segurança determina uma importante componente do custo de estocagem.

O simulador desenvolvido pode ser aplicado para o planejamento de obtenções, utilizando dados de projeção de demanda, de modo a minimizar os custos com estocagem. Entretanto, sabe-se que trabalhar em condições próximas ao ótimo, exigirá operações e processos de abastecimento cada vez mais ágeis e eficientes.

\section{REFERÊNCIAS BIBLIOGRÁFICAS}

[1] ACCIOLY, F.; AYRES, A. de P. S.; SUCUPIRA, C. Gestão de Estoques. Rio de Janeiro: Editora FGV, 2008.

[2] ABENSUR, E. O. Um modelo multiobjetivo de otimização aplicado ao processo de orçamento de capital. Gest. Prod., São Carlos, v. 19, n. 4, p. 747-758, 2012.

[3] BALLOU, R. H. Gerenciamento da Cadeia de Suprimentos: Logística Empresarial. 5. ed. Porto Alegre: Bookman, 2006.

[4] BRASIL. Índice Nacional de Preços ao Consumidor Amplo - IPCA. Portal Brasil. Disponível em: <http://www.portalbrasil.net/ipca.htm>. Acesso em: 21 de mar. 2014.

[5] BRASIL. Juros Selic. Receita Federal. Disponível em: <http:// http://www.receita.fazenda.gov.br/pagamentos/jrselic.htm>. Acesso em: 21 de mar. 2014.

[6] KRAJEWSKI, L. J.; RITZMAN, L. P.; MALHOTRA, M. K. Administração da produção e operações. 8 ed. São Paulo: Pearson/Prentice Hall, 2009

[7] MARINHA DO BRASIL. Levantamento de regras do Planejamento. Rio de Janeiro: Diretoria de Abastecimento da Marinha, 2014.

[8] MEDEIROS, J. L. Otimização com MATLAB. Rio de Janeiro: TPQB/EQ UFRJ, 2010.

[9] NETO, A. A.; LIMA, F. G.; ARAÚJO, A. M. P. Uma proposta metodológica para o cálculo do custo de capital no Brasil. R.Adm., São Paulo, v.43, n.1, p.72-83, jan./fev./mar. 2008

[10] SAMUELSON, P. A.; NORDHAUS, W. D. Economia. 18. ed. Madrid: McGraw-Hill, 2005.

[11] SLACK, N.; CHAMBERS, S.; HARLAND, C.; HARRISON, A.; JOHNSTON, R. Administração da Produção. 1 ed. São Paulo: Atlas, 2010.

[12] SILVA, R. B. Logística - Abastecimento, Distribuição e Terceirização. Rio de Janeiro: Editora FGV, 2006.

[13] TAYLOR, D. A. Logística na Cadeia de Suprimentos: uma perspectiva gerencial. São Paulo: Pearson Addison-Wesley, 2005.

[14] WANKE, P. Gestão de estoques na cadeia de suprimentos: decisões e modelos quantitativos. 3. ed. São Paulo: Atlas, 2011. 367 p. (Coleção COPPEAD de Administração).

[15] WANKE, P. Quadro conceitual para gestão de estoques: enfoque nos itens. Gest. Prod., São Carlos, v. 19, n. 4, p. 677-687, 2012. 
[16] WANKE, P. The impact of different demand allocation rules on total stock levels. Pesquisa Operacional, v. 30, n. 1, p. 33-52, 2010. 